\title{
Spinal subdural and epidural hematomas after vertebroplasty for compression fracture: a case report
}

\author{
Xudong Wang ${ }^{1} \cdot$ Yan Peng ${ }^{1} \cdot$ Jincheng Qiu ${ }^{1}$ - Dongsheng Huang ${ }^{1}$
}

Received: 25 July 2018 / Accepted: 30 August 2018

(c) International Spinal Cord Society 2018

\begin{abstract}
Introduction Vertebroplasty (VP) is a well-known and effective therapeutic method for relieving the pain and improving the quality of life of patients with vertebral compression fractures. Major complications of VP are infrequent, occurring in no more than $1 \%$ of such procedures, whereas spinal subdural and epidural hematomas are extremely rare complications.

Case Presentation We present a unique case of a spinal subdural hematoma (sSDH) (from T4 to T12) occurring immediately after VP to treat a traumatic vertebral compression fracture, followed by a spinal epidural hematoma (SEH) (from T3 to T12) after the decompressive laminectomy, occurring in a 64-year-old patient. After removing of the big spinal epidural hematoma, the patient's symptoms improved progressively and she recovered after 2 months.

Discussion This is a rare case of SSDH and SEH occurring continuously after VP. The pathogenesis may be that the puncture damaged the abnormal blood vessel of spinal dura mater. A small-probability event is not an impossible event. We hope to bring more attention to the rare complications of VP by sharing this unusual case.
\end{abstract}

\section{Introduction}

Since Galibert et al. [1] first performed vertebroplasty (VP) in 1987, it has become a common therapeutic method for the treatment of painful vertebral angiomas, osteoporotic vertebral fractures, compression fractures, and other lesions. The VP procedure involves injecting an appropriate amount of the polymethyl methacrylate (PMMA) close to the fractured vertebral body in order to enhance the stability of the spine [2]; this can relieve pain and improve the patient's quality of life. Although the success rate of the VP procedure exceeds $90 \%$, it can also lead to major and severe complications such as spinal cord compression, adverse reactions caused by the bone cement, epidural and subdural hematomas, pulmonary emboli, infection, and death [3, 4]. Major complications of VP are infrequent, occurring in no more than $1 \%$ of such procedures. The vast majority of the

These authors contributed equally: Xudong Wang, Yan Peng.

Dongsheng Huang

hdongsh@mail.sysu.edu.cn

1 Department of Orthopedics, Sun Yat-sen Memorial Hospital of Sun Yat-sen University, Guangzhou, Guangdong, China complications are attributable to migration of the bone cement into the veins, which can result in the pulmonary embolism [5], or spilling of the bone cement spill into the spinal canal, resulting in the spinal cord compression [6].

Spinal subdural hematoma (sSDH) and spinal epidural hematoma (SEH) are extremely rare complications that usually develop within a few hours of the procedures. Most sSDHs occur at the lower thoracic region and usually extend in length from two to five segments [7]. Most SEHs occur on the spinal cord posteriorly, whereas most sSDHs are located on the spinal cord anteriorly [8]. As far as we know, only five cases about SSDH after VP have thus far been mentioned in literature [9-12], where no SEHs have occurred after decompressive laminectomy. We report the first case of a sSDH (from T4 to T12) occurring immediately after VP for a traumatic vertebral compression fracture followed by a SEH (from T3 to T12) after the decompressive laminectomy. We also discuss the possible pathogenesis of sSDH and SEH.

\section{Case presentation}

Our patient, a 64-year-old woman, complained of lower thoracic pain after a fall on the ground. After admission to the hospital, she underwent magnetic resonance imaging 


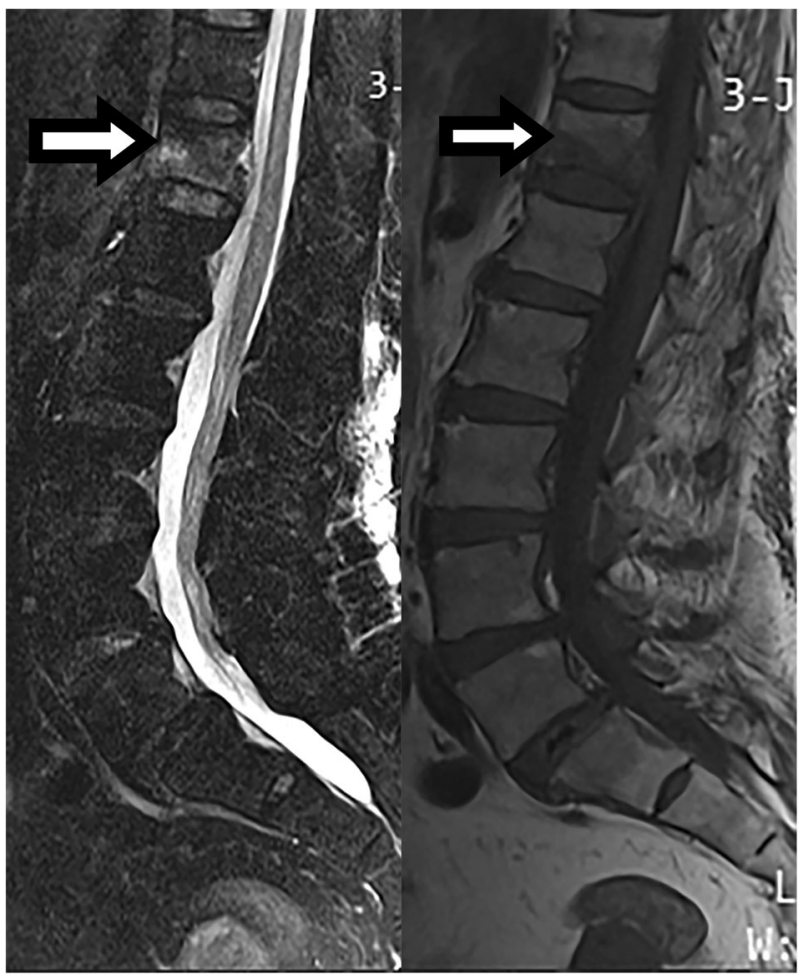

Fig. 1 Magnetic resonance imaging of the thoracic spine pre-percutaneous vertebroplasty demonstrating a T12 compression fracture

(MRI) of the thoracic spine, which revealed a T12 compression fracture (Fig. 1). Subsequently a percutaneous vertebroplasty (PVP) was performed under local anesthesia. After the operation was completed, the patient complained of weakening of muscle strength (strength $3+/ 5$ ), hypoesthesia, and dysesthesia in the right leg. After half an hour, she presented with further weakening of muscle strength (strength 2/5), hypoesthesia, and dysesthesia of both lower extremities as well as abdominal zonesthesia. An immediate MRI scan demonstrated a high-signal aggregation in front of the spinal cord from T4 to T12, which appeared to be causing the spinal cord compression (Fig. 2). Owing to concern regarding the acute decrease of muscle strength in both lower extremities, the patient immediately underwent a decompressive laminectomy from $\mathrm{T} 3$ to $\mathrm{T} 12$, where the spinal canal was obviously compressed. A hematoma but no epidural hemorrhage was then found in the subdural spinal space. Finally, a durotomy was performed in order to protect the spinal cord. However, to avoid damaging the spinal cord, the hematoma wasn't removed due to the stenosis of the thoracic spinal canal. After the operation, the patient's symptoms improved progressively for several days, after which they again worsened. The postoperative MRI confirmed the presence of a large hematoma behind the spinal cord from T3 to T12, which was causing the spinal cord compression (Fig. 3).

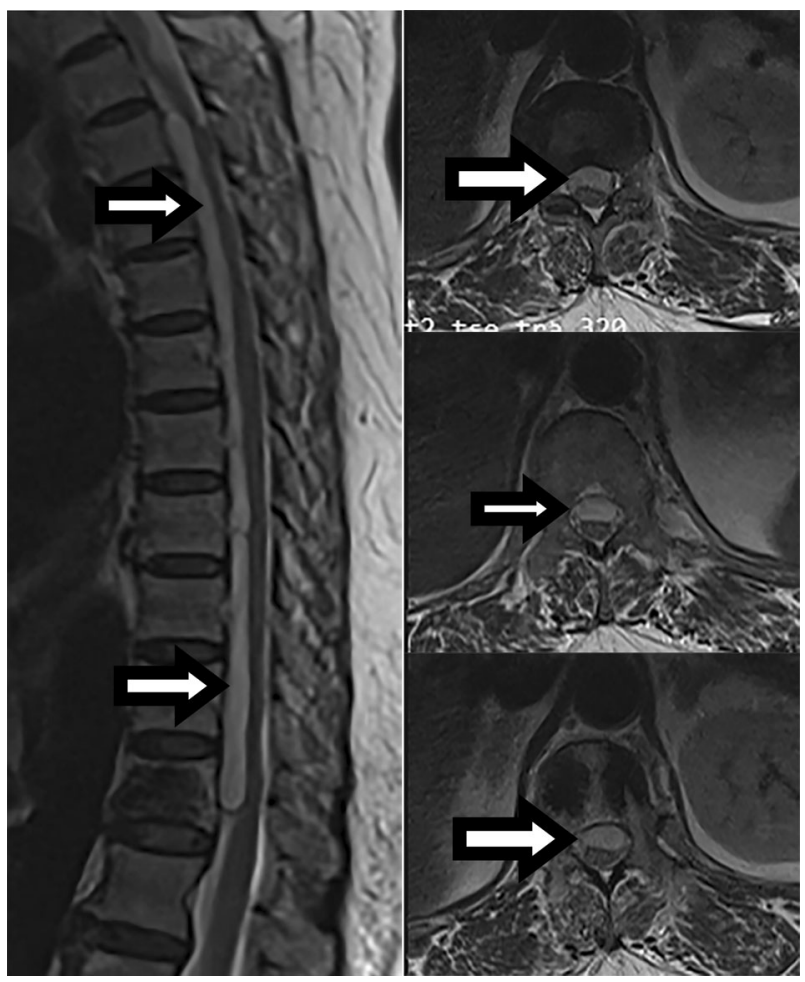

Fig. 2 Magnetic resonance imaging post-percutaneous vertebroplasty demonstrating a high-signal aggregation in front of the spinal cord from $\mathrm{T} 4$ to $\mathrm{T} 12$, indicating spinal cord compression

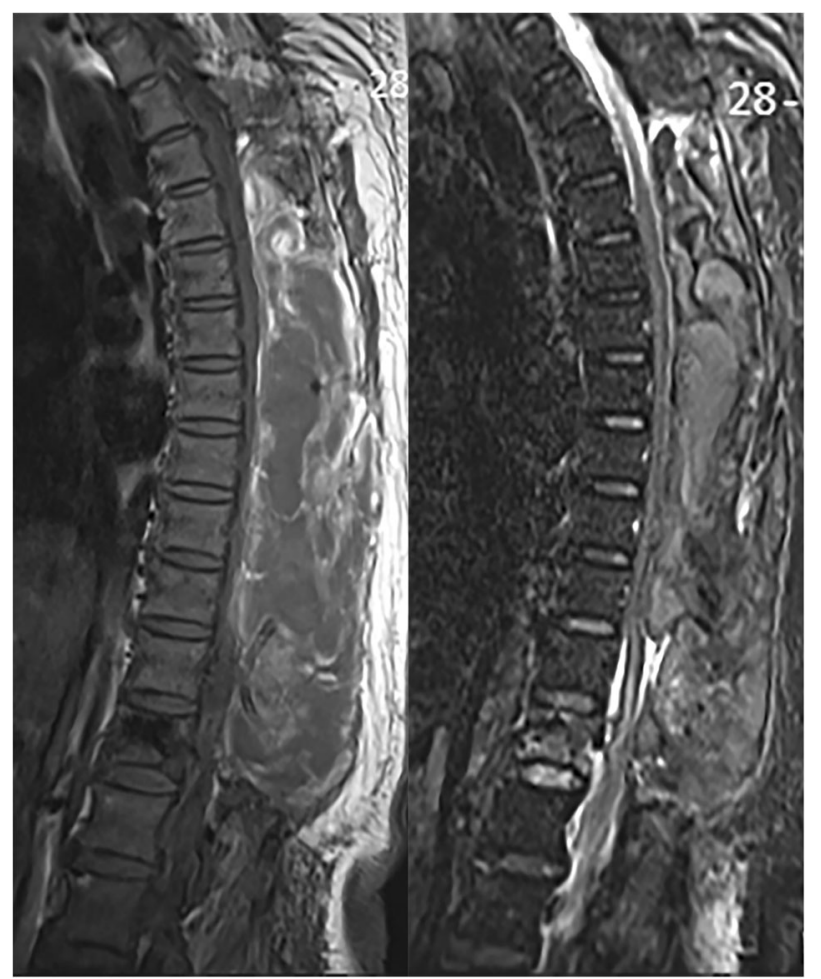

Fig. 3 Magnetic resonance imaging post-decompressive laminectomy confirming the presence of a large hematoma behind the spinal cord from T3 to T12, which was causing spinal cord compression 


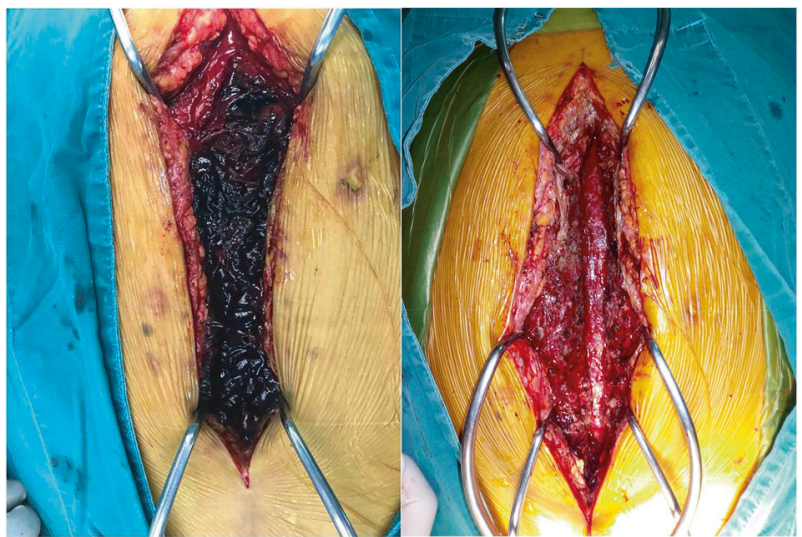

Fig. 4 The photographs during the surgery demonstrating a big spinal epidural hematoma (from T3 to T12) behind the spine (the left photograph), and the spinal cord was fully decompressed after surgical removal of the hematoma (the right photograph)

At this point the patient was transferred to our department, where a physical examination revealed a weakening of muscle strength (strength 3/5) in the right leg, hyperreflexia of the lower extremity, and a positive Babinski sign on both sides. The hematology and coagulation tests of the patient were normal. After surgical contraindications had been ruled out, she was referred for evacuation of hematoma. Then the big spinal epidural hematoma was removed during the surgical procedure (Fig. 4). After surgery, the postoperative MRI scan demonstrated adequate removal of the large hematoma and surgical decompression of the spinal cord (Fig. 5). After rehabilitation and physical therapy, the patient progressively recovered both muscle strength and sensation in the right leg. After 2 months, her muscle strength had recovered fully (strength 5/5), as had sensory function in the right lower extremity.

\section{Discussion}

The numbers of vertebral compression fractures have gradually increased because of the aging population and changing lifestyles. VP has been a prevalent therapeutic method for the treatment of painful vertebral angiomas, osteoporotic vertebral fractures, compression fractures, and other lesions. In the course of time, as the indications for VP have expanded, vertebral compression fracture due to acute trauma has become one such indication. Although VP is regarded as an extremely safe operation, major complications are still often seen. Among these, $\mathrm{SSDH}$ and SEH are extremely rare. As far as we know, this is the first case of a sSDH (from T4 to T12) occurring immediately after VP to treat a traumatic vertebral compression fracture, followed by a SEH (from T3 to T12) after the decompressive laminectomy.

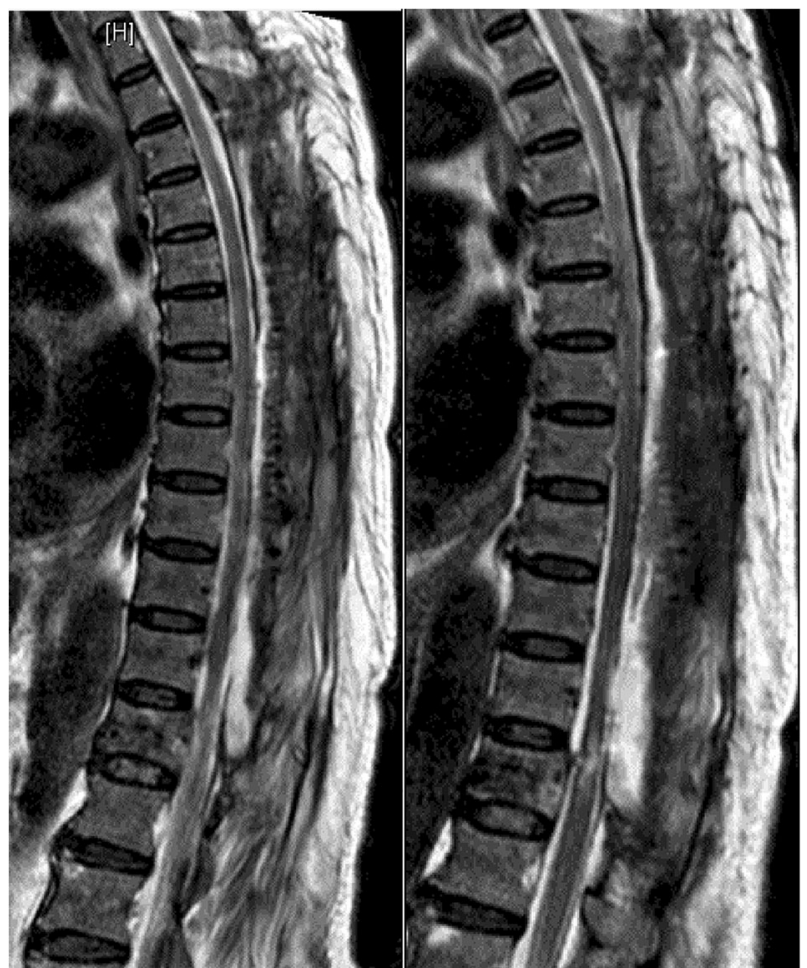

Fig. 5 Magnetic resonance imaging demonstrating adequate removal of a large hematoma and surgical decompression of the spinal cord

The pathogenesis of spinal SDH is still unknown, although rupture of subdural bridging veins is deemed to be the main cause in intracranial SDH. However, the spinal subdural space differs from the intracranial subdural space in that bridging veins are not found in the latter [13]. The possible mechanism of our case would be that puncture of the spinal dura mater could lead to sSDH due to trauma to an abnormal blood vessel of spinal dura mater.

The possible pathogenesis of SEH in our case may stem from the longitudinal durotomy. After the procedure, spinal subdural blood would have flowed into the spinal epidural space, resulting in a massive hematoma behind the spinal cord and hence its compression.

We usually consider VP to be a safe operation. However, its possible complications should not be ignored. First, in order to reduce the number of such complications, the indications for VP should not be expanded. Second, preoperative examinations should be performed routinely, and emergency PVP surgery is not recommended. Third, the operation must be performed by experienced doctors following standardized surgical procedures. Last, biplanar fluoroscopic guidance during VP procedures should be performed carefully and slowly, thus making VP safer. Before the surgical procedure, its potential complications ought to be well known and, if necessary, emergent treatment implemented. 


\section{Conclusion}

We present a rare case of a $\mathrm{sSDH}$ (from $\mathrm{T} 4$ to $\mathrm{T} 12$ ) occurring immediately after VP for a traumatic vertebral compression fracture, followed by a SEH (from T3 to T12) after the decompressive laminectomy. The pathogenesis of this complication may be that the puncture damaged the abnormal blood vessel of spinal dura mater. In sum, because a small-probability event is not an impossible event, more attention ought to be given to the major potential complications of VP. In short, this procedure must be performed by experienced surgeons following standardized surgical procedures-surgeons who can recognize potential risk factors and thus make VP safer.

Acknowledgements We would like to thank the patient for allowing us to present this case and thank LetPub (www.letpub.com) for providing linguistic assistance during the preparation of this manuscript.

Funding This research did not receive any specific grant from funding agencies in the public, commercial, or not-for-profit sectors.

\section{Compliance with ethical standards}

Conflict of interest The authors declare that they have no conflict of interest.

Consent The patient has consented to submission of this case report to the journal.

\section{References}

1. Galibert P, Deramond H, Rosat P, Le Gars D. Preliminary note on the treatment of vertebral angioma by percutaneous acrylic vertebroplasty. Neurochirurgie. 1987;33:166-8.
2. Blasco J, Martinez-Ferrer A, Macho J, San Roman L, Pomes J, Carrasco J, et al. Effect of vertebroplasty on pain relief, quality of life, and the incidence of new vertebral fractures: a 12-month randomized follow-up, controlled trial. J Bone Miner Res. 2012;27:1159-66.

3. Moreland DB, Landi MK, Grand W. Vertebroplasty: techniques to avoid complications. Spine J. 2001;1:66-71.

4. Vats HS, McKiernan FE. Infected vertebroplasty: case report and review of literature. Spine (Phila Pa 1976). 2006;31: E859-62.

5. Venmans A, Lohle PN, van Rooij WJ, Verhaar HJ, Mali WP. Frequency and outcome of pulmonary polymethylmethacrylate embolism during percutaneous vertebroplasty. Am J Neuroradiol. 2008;29:1983-5.

6. Chotivichit A, Korwutthikulrangsri E, Churojana A, Songsaeng D. Complications in vertebroplasty. J Med Assoc Thai. 2012;95 (Suppl):S75-81.

7. Kreppel D, Antoniadis G, Seeling W. Spinal hematoma: a literature survey with meta-analysis of 613 patients. Neurosurg Rev. 2003;26:1-49.

8. Braun P, Kazmi K, Nogues-Melendez P, Mas-Estelles F, ApariciRobles F. MRI findings in spinal subdural and epidural hematomas. Eur J Radiol. 2007;64:119-25.

9. Cosar M, Sasani M, Oktenoglu T, Kaner T, Ercelen O, Kose KC, et al. The major complications of transpedicular vertebroplasty. $\mathrm{J}$ Neurosurg Spine. 2009;11:607-13.

10. Mattei TA, Rehman AA, Dinh DH. Acute spinal subdural hematoma after vertebroplasty: a case report emphasizing the possible etiologic role of venous congestion. Glob Spine J. 2015;5:e52-8.

11. Lee KD, Sim HB, Lyo IU, Kwon SC, Park JB. Delayed onset of spinal subdural hematoma after vertebroplasty for compression fracture: a case report. Korean J Spine. 2012;9:285-8

12. Tropeano MP, La Pira B, Pescatori L, Piccirilli M. Vertebroplasty and delayed subdural cauda equina hematoma: review of literature and case report. World J Clin Cases. 2017;5:333-9.

13. Ahn ES, Smith ER. Acute clival and spinal subdural hematoma with spontaneous resolution: clinical and radiographic correlation in support of a proposed pathophysiological mechanism. Case report. J Neurosurg. 2005;103:175-9. 